\title{
The Impact of Link Error Modeling on the Quality of Streamed Video in Wireless Networks
}

\author{
Wolfgang Karner, Olivia Nemethova, Markus Rupp \\ Institute of Communications and Radio-Frequency Engineering \\ Vienna University of Technology, Austria \\ Gusshausstrasse 25/389, A-1040 Vienna, Austria \\ Email: \{wkarner, onemeth, mrupp\}@nt.tuwien.ac.at
}

\begin{abstract}
The influence of channel error characteristics on higher layer protocols or methods which are considering or even exploiting the error statistics is significant especially in wireless networks where fading and interference effects result in error pattern correlation properties (error bursts). In this work we are analysing the impact of the channel properties directly on the quality of streamed video. We are focusing on the quality of transmitted H.264/AVC video streaming over UMTS DCH (Dedicated Channel) and compare the quality of the streamed video simulated over measured link error traces (the measurements performed in a live UMTS network) to simulations with a memoryless channel and to models with enhanced error characteristics. The results show that appropriate modeling of the link layer error characteristics is very important but it can also be concluded that the error correlation properties of the link- or the network-layer model do not have an impact on the quality of the video stream as long as the resulting IP packet error probability remains unchanged.
\end{abstract}

\section{INTRODUCTION}

In most communication channels there is a certain small probability of having transmission errors. Other than in wired links, wireless channels are prone to have even more errors due to fading effects or interference which furthermore add correlation properties (memory) to the channel characteristics resulting in error bursts. Beginning in the 1960s with the work of Gilbert [1], there is still lots of effort in research today for finding appropriate models with particular error characteristics. In [1] a two-state Markov model with one 'good' and one 'bad' state was proposed, where no errors occur in the 'good' state but in the 'bad' state the error probability takes some value $>0$. Enhancements to that model can be found in the work of Elliot [2] where errors can occur also in the 'good' state and in Fritchman [3] which makes use of more than only one 'good' state. Others are modeling the error characteristics by using further Hidden Markov Models (HMM) [4] [5] or even some Markov modulated renewal processes for meeting particular properties of the wireless links [6].

The error characteristics of the channel and thus also the model properties have a great impact on the performance of methods and algorithms (e.g. for cross-layer optimization of wireless systems) which are considering [7] or even exploiting [8] the error characteristics of the underlying channels. In [9] it was shown that there is also a strong impact of the secondorder error statistics of the channel onto the performance of the higher layer protocols.
In this work we are investigating the impact of link error modeling directly on the quality of streamed video in wireless networks. We are focusing on H.264/AVC video streaming [10] over UMTS DCH (Dedicated CHannel).

In literature, lots of different link error models are used for simulating video streaming over wireless networks. E.g. in [11] a memoryless error model was used for evaluating video streaming over UMTS DCH, motivated by simulation results in [12] from which it was concluded that the assumption of uncorrelated block errors is valid in UMTS networks. However, the assumption of uncorrelated block errors contradicts measured results presented in [6]. Others (e.g. [13]) are using a special case of Gilbert's model [1] representing a two-state Markov chain which is uniquely specified by the average burstlength [14] and the packet loss rate for modeling the link error characteristics.

For the investigation of the impact of link error modeling on the resulting quality of the video stream we are comparing the link- and network-layer characteristics as well as the resulting video quality simulated with various link error models. Referring to the models used in the above mentioned literature we have been using a memoryless channel, the special case of Gilbert's model and a Markov modulated Weibull renewal process ('Karner - two-layer' model presented in [6]). We are also comparing the results from simulations using these models with simulations with measured link layer error traces from measurements performed in a live UMTS network in the city center of Vienna, Austria.

The results of the comparison show that the higher order statistics of the link errors do have a significant impact on the quality of the streamed video (analysed via statistics of Y-PSNR per frame) due to the difference in resulting network layer error probability. In contradiction to that there is no impact of the error correlation properties in the network layer (burst statistics) on the average video quality as long as the resulting IP packet error probability remains the same. This is due to the error propagation when using predictive coding in the streamed video sequence. These results are in contradiction to the results of [15] where it was shown that longer error bursts lead to less video quality.

This document is organized as follows. In Section II the models compared in this document are introduced and their characteristics are shown in detail. Section III presents the 
experimental setup also explaining the protocol stack for streaming video over the packet switched (PS) domain of UMTS. The impacts of modeling the link errors in the various ways on the video quality are demonstrated in Section IV for the video stream encoded by the H.264/AVC video codec. Section V provides a summary and conclusions.

\section{Characteristics OF COMPARED Models}

As already mentioned, for simulating the link error characteristics, additionally to the measured link error trace we have been using a memoryless channel, the special case of Gilbert's model (two-state Markov chain) and a Markov modulated two-state Weibull renewal process ('Karner - two-layer'). The measured error trace was taken from measurements performed in a live UMTS network in Vienna, Austria [6]. Although all of the models are producing the same link error probability $(0.266 \%)$ as measured in the live UMTS network, they are showing significant differences in their error characteristics. That can be observed in Figs. 1 and 2, where the CDFs of the number of error free link layer packets between two errors (gaplength) and the CDFs of the number of subsequently received errors (burstlength) are presented.

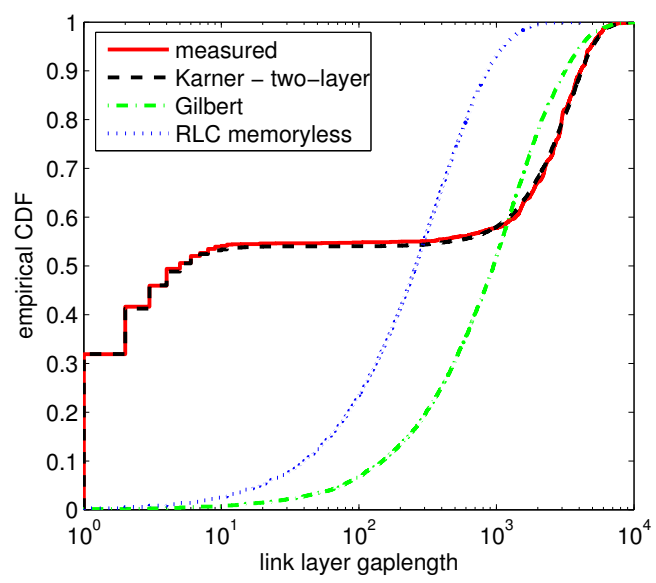

Fig. 1. Comparison of the link layer gaplengths.

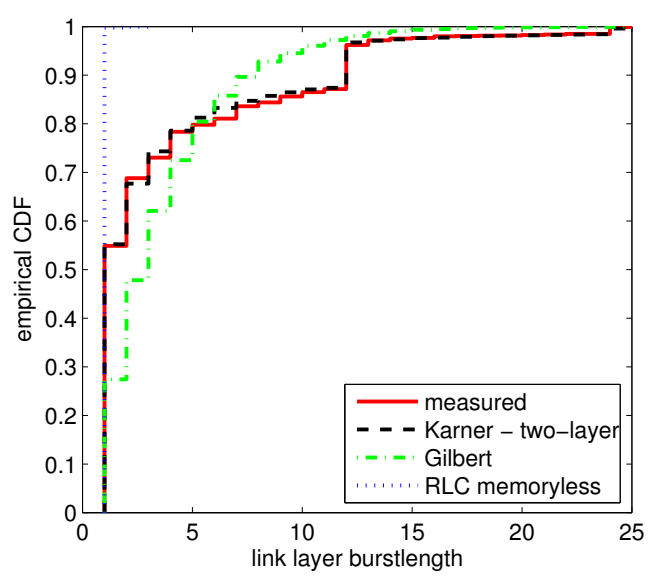

Fig. 2. Comparison of the link layer burstlengths.

\section{EXPERIMENTAL SETUP}

\section{A. Protocol Architecture}

For transmission of a video stream over the UMTS network the following procedure of packetization has to be performed. Each frame of the video is first subdivided into smaller parts (slices) which then are encoded. Encoded video slices are encapsulated into RTP (Real Time Protocol) packets and Fig. 3 shows how the RTP packets are further processed by underlaying protocol layers [16], [17]. Each RTP packet is

2

\begin{tabular}{|l|l|}
\hline RLC payload & RLC payload \\
\hline
\end{tabular}

3

$\mathrm{H}$

4

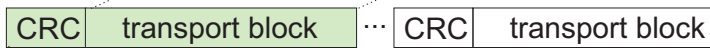

5

channel coded block $\mathrm{T}$

6 TTI

Fig. 3. Packetization example of a video slice for transmission over UMTS radio interface: 1 - IP packet, 2 - RLC segmentation, 3 - RLC and MAC header addition, 4 - CRC addition, 5 - transport block concatenation and channel coding (with tailing bits addition), 6 - after rate matching and interleaving over one TTI.

encapsulated into a UDP and further on into an IP packet. Then the UTRAN (UMTS Terrestrial Radio Access Network) RLC (Radio Link Control) layer performs segmentation of the IP packets and adds an RLC header. After the mapping of the packets onto the transport channel, performed by the MAC (Medium Access Control) layer, the RLC payload in connection with the RLC and MAC headers becomes a transport block (TB). For packet switched bearers the RLC of UTRAN can work in acknowledged mode (AM) offering RLC packet retransmissions or in unacknowledged mode (UM) enabling only error detection but no feedback.

After attaching CRC bits [18] to the transport blocks, these are segmented/concatenated into code blocks and the bitstream is encoded by a channel code. For packet oriented applications usually turbo coding is used with a coding rate of $1 / 3$, which can further be punctured to match the rate with the physical resources.

Before mapping the bitstream onto the physical channels for transmission, a 1st interleaving over one TTI (Transmission Timing Interval), radio frame segmentation, transport channel multiplexing, physical channel segmentation and a 2nd interleaving (over one radio frame) is performed.

The analysis for this work has been performed with a UMTS DCH in DL (Down Link) with 384kbit/s. We have been using IPv4, a RLC payload of 320 bits and 16 bits of CRC. The TTI was $10 \mathrm{~ms}$ and there have been transmitted $12 \mathrm{TBs}$ within 
each TTI by using a spreading factor of eight. Before the segmentation of an IP packet, the Packet Data Convergence Protocol (PDCP) may perform header compression which was not used in our case.

\section{B. Simulation of H.264 Encoded Video over Error Prone Links}

In Fig. 4 our experimental setup can be seen. We adapted the Joint Model (JM) H.264/AVC [19] to our needs by adding the interface for the IP error traces and by implementing a simple error concealment scheme (for I frames we use weighted averaging [20] and for P frames we copy the corresponding location from the previous frame) at the decoder. The encoder is modified to deliver the IP packet lengths for mapping the IP packets onto the link layer packet trace.

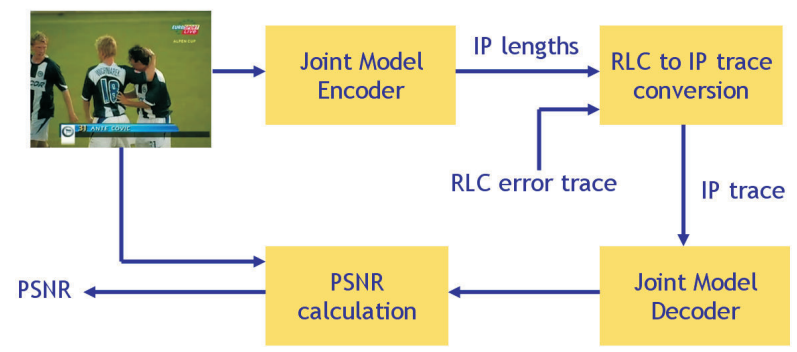

Fig. 4. Scheme of the experimental setup.

For our experiments we selected a 'soccer' video sequence with SIF $(\mathrm{QVGA})$ picture resolution $(320 \times 240$ pixel $)$ and a frame rate of 10 , containing a soccer match with different scenes. We encoded the sequence using I and P frames only (every 40th frame is an I frame). We chose the baseline profile with slicing mode two with $\leq 750$ bytes per slice. The quantization parameter was set to 26 and the rate-distortion optimization was disabled. To obtain reliable results, the video was decoded several times resulting in $\approx 10$ hours of video stream.

\section{RESULTS}

To evaluate the end-to-end video quality, we use the peak to signal-to-noise ratio of the luminance component (Y-PSNR) given for the $n$th luminance frame $\mathbf{Y}_{n}$ by

$$
\begin{aligned}
\operatorname{Y-PSNR}(n) & =10 \cdot \log _{10} \frac{255^{2}}{\operatorname{MSE}(n)}, \\
\operatorname{MSE}(n) & =\frac{1}{N \cdot M} \sum_{i=1}^{N} \sum_{j=1}^{M}\left[\mathbf{Y}_{n}(i, j)-\mathbf{F}_{n}(i, j)\right]^{2},
\end{aligned}
$$

where $\operatorname{MSE}(n)$ denotes the mean square error of the $n$th luminance frame $\mathbf{Y}_{n}$ compared to the luminance frame $\mathbf{F}_{n}$ of the reference sequence with the non-compressed original (non-degraded) sequence as a reference. The resolution of the frame is $N \times M$, indexes $i$ and $j$ address particular luminance values within the frame.

In Fig. 5 histograms of the Y-PSNR per frame for the considered models are presented. It can be observed that

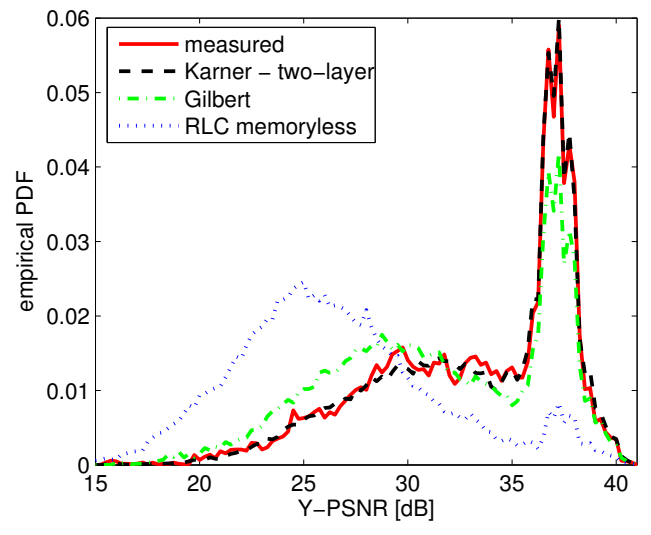

Fig. 5. Y-PSNR per frame for the various link layer error models.

despite having the same link layer BLER (Block Error Ratio) (see Table I) only the 'Karner - two-layer' model meets the measured statistics. This is due to the fact that only the 'Karner - two-layer' model shows almost the same link error characteristics as the measured traces. All the other models and especially the memoryless channel have completely different statistics of the burst- and gaplengths and thus result in a different number of erroneously received link layer packets (TBs - Transport Blocks) within one IP packet (see Fig. 6). This in turn means a much higher IP BLER e.g. in case of the memoryless link layer characteristics as shown in Table I. From these results we conclude that it is important to have the correct higher order statistics when modeling the link layer for video streaming simulations.

TABLE I

COMPARISON OF LINK-LAYER AND IP BLER (BLOCK ERROR RATIO).

\begin{tabular}{|c|c|c|}
\hline Model & link layer BLER [\%] & IP BLER [\%] \\
\hline \hline measured & 0.266 & 0.888 \\
\hline Karner - two-layer & 0.262 & 0.892 \\
\hline Gilbert & 0.266 & 1.009 \\
\hline RLC memoryless & 0.266 & 4.501 \\
\hline
\end{tabular}

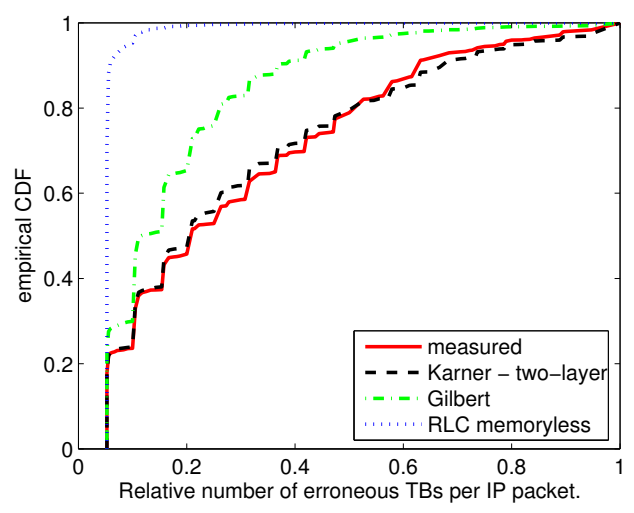

Fig. 6. Relative number of erroneous TBs per (erroneous) IP packet.

Contrary to the conclusions for the link layer modeling it 
will be shown in the following that for modeling of the errors in the network layer it is not important to meet the correct higher order statistics for evaluating the quality of streamed video with common parameter settings. In Fig. 7 we can see that by applying a memoryless channel model in the network layer with appropriate IP BLER $(0.888 \%$ instead of $4.501 \%$ - see Table I), the measured statistics of the streamed video quality (Y-PSNR per frame) is met with a memoryless model as well, despite having completely different network layer error characteristics.

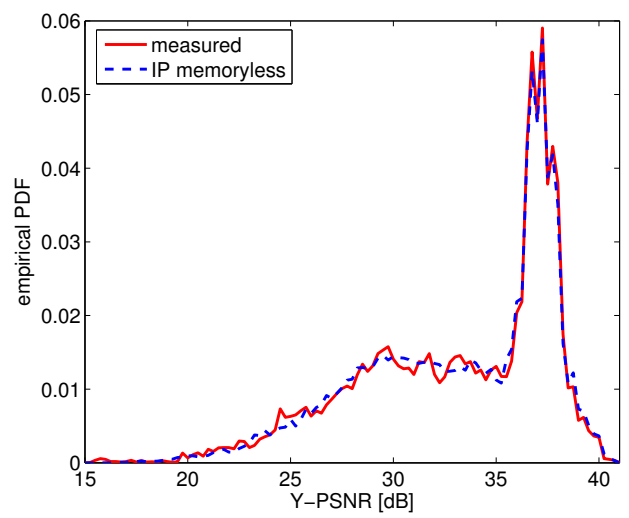

Fig. 7. Y-PSNR per frame for IP memoryless model vs. measurements.

These differences in the network layer error characteristics can be seen in Figs. 8 and 9, where the PDFs of the IP burstlengths (number of subsequently erroneous IP packets) and the PDFs of the IP gaplengths (number of error-free IP packets between two errors) are presented.

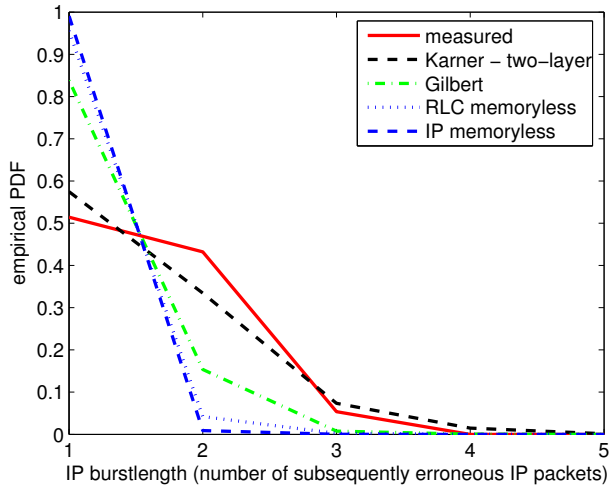

Fig. 8. Comparison of IP burstlengths for the various error models.

The fact that the different statistics of the IP burst- and gaplengths of the measured traces and the memoryless network layer model are resulting in the same video streaming quality is becoming even more interesting when observing the difference in the relative number of erroneous IP packets per (erroneous) video frame in Fig. 10. These results are in contradiction to [15] where lower video quality for longer IP error bursts and thus also more erroneous IP packets per video frame is expected.

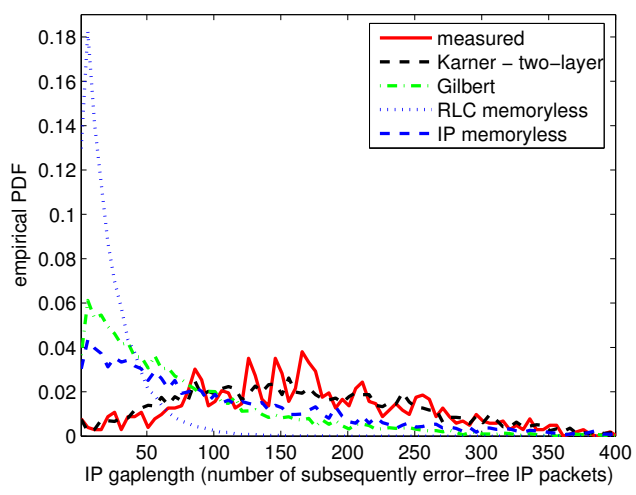

Fig. 9. Comparison of IP gaplengths for the various error models.

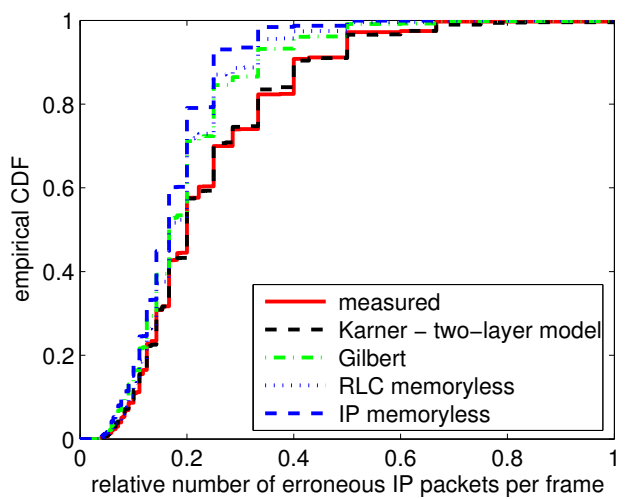

Fig. 10. Relative number of erroneous IP packets per video frame.

As presented in Section II/B, the evaluation of the video quality was performed using H.264/AVC with a common parameter setting for video streaming over wireless networks, meaning the video stream consists of one I and 39 following $\mathrm{P}$ frames where the latter ones are predictively encoded. This predictive coding of the $\mathrm{P}$ frames leads to error propagation within one GOP (Group of Pictures) which in turn is the reason for having the same video quality with different IP burstlengths and also with a different number of erroneous IP packets per video frame.

When encoding the video with I frames only, there is no error propagation in the decoded video sequence. As a consequence a difference in the Y-PSNR values per frame between the memoryless network layer and the measured characteristics can be observed as shown in Fig. 11. There, PDFs of the Y-PSNR values per frame - only the part of the erroneous frames - are presented. Note that there is a higher peak in the PDF for the error-free frames at higher Y-PSNR values.

Thus, our conclusion is that with a memoryless channel model in the IP layer (equivalently a memoryless channel model in the link layer with appropriate BLER), a plain IP-UDP-RTP protocol stack above RLC layer and common parameter settings for video streaming over UMTS DCH, despite having different higher order statistics in the network 


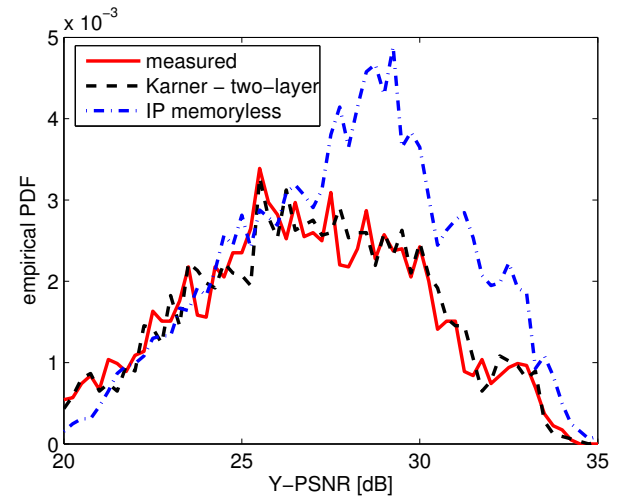

Fig. 11. Y-PSNR per frame for only I frames (no error propagation).

layer, the statistical end-to-end quality of streamed video data will be the same as for channels with highly correlated errors. Therefore, when modeling the link layer, special attention has to be paid to the error correlation properties even with plain IP-UDP-RTP protocol stack, while a model in the network layer just has to meet the correct packet error probability.

Of course, it is important to mention that our analysis is only based on time independent Y-PSNR statistics and thus different results for the perceived end user quality may be expected.

\section{Summary AND CONCLUSIONS}

It is well known that the higher order statistics of the link errors are heavily influencing special methods or algorithms in the higher protocol layers which are considering or even exploiting the link error characteristics. Thus, for evaluation purposes of these special algorithms in the higher layers or equivalently for the evaluation of the end-to-end quality of the services it is important to use models of the link layer which are presenting the correct link error characteristics. In this document we are investigating the impact of the link layer modeling directly on the quality of streamed H.264/AVC video sequences with common parameter settings for video streaming over UMTS and a plain IP-UDP-RTP protocol stack. We are comparing the link- and network-layer characteristics and also the resulting video quality in Y-PSNR values of various link layer error models ranging from a memoryless model to a model which is capable of meeting the higher order statistics of the link errors almost perfectly. We have also compared the results of the simulations with these models to the results obtained by using measured link error traces from measurements performed in live UMTS networks in the city center of Vienna, Austria. The results show that it is very important to model the correlation properties of the link errors appropriately in order to meet the measured video quality. On the other hand we also present in this document that with a memoryless channel model in the IP layer (equivalently a memoryless channel model in the link layer with appropriate packet error probability), despite having different higher order statistics in the network layer, the statistical end-to-end quality of streamed video data will be the same as for channels with highly correlated errors. We show in this paper that this is due to the error propagation in the video when using predictive coding in the video sequences. When using the rather unusual setting in the video coder of having only I frames and thus no error propagation, the influence of the error correlation properties becomes significant. The impact will be even more relevant when considering higher error probabilities in the link or network layer.

\section{ACKNOWLEDGEMENTS}

We thank mobilkom austria AG\&CoKG for technical and financial support of this work. The views expressed in this paper are those of the authors and do not necessarily reflect the views within mobilkom austria AG\&CoKG.

\section{REFERENCES}

[1] E.N. Gilbert, "Capacity of a burst-noise channel," Bell Systems Technical Journal, vol. 39, pp. 1253-1265, Sept. 1960.

[2] E.O. Elliot, "Estimates of error rates for codes on burst-noise channels," Bell Systems Technical Journal, vol. 42, pp. 1977-1997, Sept. 1963.

[3] B.D. Fritchman, "A Binary Channel Characterization Using Partitioned Markov Chains," IEEE Trans. Information Theory, vol. 13, no. 2, pp. 221-227, Apr. 1967.

[4] W. Turin, Digital Transmission Systems: Performance Analysis and modelling. New York: McGraw-Hill, 1999.

[5] L.R. Rabiner, B.H. Juang, "An Introduction to Hidden Markov Models," IEEE ASSP Magazine, Vol. 3, pp. 4-16, Jan. 1986.

[6] W. Karner, M. Rupp, "Measurement based Analysis and Modelling of UMTS DCH Error Characteristics for Static Scenarios," in Proc. of 8th International Symposium on DSP and Communication Systems 2005 (DSPCS'2005), Sunshine Coast, Australia, December 2005.

[7] O. Nemethova, W. Karner, A. Al-Moghrabi, M. Rupp, "Cross-Layer Error Detection for H.264 Video over UMTS," in Proc. of Wireless Personal Multimedia Communications 2005 (WPMC 2005), Denmark, Sept. 2005.

[8] W. Karner, O. Nemethova, P. Svoboda, M. Rupp, "Link Error Prediction Based Cross-Layer Scheduling for Video Streaming over UMTS," in Proc. of the 15th IST Mobile \& Wireless Communications Summit 2006, Myconos, Greece, June 2006.

[9] M. Zorzi, R.R. Rao, "Perspectives on the Impact of Error Statistics on Protocols for Wireless Networks," IEEE Personal Communications, Vol. 6, pp. 32-40, Oct. 1999.

[10] T. Wiegand, G.J. Sullivan, G. Bjontegaard,A. Luthra, "Overview of the H.264/AVC Video Coding Standard," IEEE Transactions on Circuits and Systems for Video Technology, vol. 13, no. 7, pp. 560-576, July 2003.

[11] A. Lo, G. Heijenk, I. Niemegeers, "Evaluation of MPEG-4 Video Streaming over UMTS/WCDMA Dedicated Channels," in Proc. of the First IEEE International Conference on Wireless Internet, 2005.

[12] M.C. Necker, S. Saur, "Statistical Properties of Fading Processes in WCDMA Systems," in Proc. of the 2nd International Symposium on Wireless Communication Systems (ISWCS 2005), pp.54-58, Sept. 2005.

[13] Qi Qu, Y. Pei, J.W. Modestino, X. Tian, "Source-Adaptive FEC/UEP Coding For Video Transport Over Bursty Packet Loss 3G UMTS Networks: A Cross-Layer Approach," 60th IEEE Vehicular Technology Conference (VTC2004-Fall), Vol. 5, pp.3150-3154, Sept. 2004.

[14] ITU-T Rec. M.60, 3008; ITU-T Rec. Q.9, 0222.

[15] Y.J. Liang, J.G. Apostolopoulos, B. Girod, "Analysis of Packet Loss for Compressed Video: Does Burst-Length Matter?" in Proc. of IEEE International Conference on Acoustics, Speech and Signal Processing (ICASSP'03), Vol. 5, pp.684-7, 2003.

[16] 3GPP TR 25.944, "Channel coding and multiplexing examples," v.4.1.0, June 2001.

[17] 3GPP TR 25.322, "Radio Link Control (RLC) protocol specification," v.4.12.0, June 2004.

[18] 3GPP TR 25.212, "Multiplexing and channel coding (FDD)," v.4.6.0, October 2002.

[19] H.264/AVC Software Coordination, "Joint Model Software," ver.10.1, available in http://iphome.hhi.de/suehring/tml/.

[20] M.T. Sun, A.R. Reibman, "Compressed Video over Networks," Signal Processing and Comm. Series, Marcel Dekker Inc., New York, 2001. 